\title{
Nonlinear Differential Problem with p-Laplacian and via Phi-Hilfer Approach: Solvability and Stability Analysis
}

\author{
Hamid Beddani ${ }^{1, *}$, Moustafa Beddani ${ }^{2}$, Zoubir Dahmani ${ }^{3}$ \\ ${ }^{1}$ Laboratory of Complex Systems of the Higher School of Electrical and Energy Engineering of Oran, \\ 31000, Algeria \\ beddanihamid@gmail.com \\ ${ }^{2}$ Department of Mathematics, University of Sidi Bel-Abbès 22000, Algeria \\ beddani2004@yahoo.fr \\ ${ }^{3}$ Laboratory of Pure and Applied Mathematics, Abdelhamid Bni Badis University, 27000, Algeria \\ zzdahmani@yahoo.fr \\ *Correspondence: beddanihamid@gmail.com
}

\begin{abstract}
AвSTRACT. This paper we consider a study of a general class of nonlinear singular fractional DEs with p-Laplacian for the existence and uniqueness solution and the Hyers-Ulam (HU) stability. result via $\varphi$-Hilfer derivative is studied. Then, an existence of one solution is investigated. Some illustrative examples are discussed at the end.
\end{abstract}

\section{INTRODUCTION}

Recently, fractional differential equations with boundary conditions are being studied by many interested people. This is because fractional differential equations describe many more real operations than classical differential equations. Therefore, partial differential equations appear in many engineering and technological disciplines that include several sciences; See for example $[1,3-6,8,17,18,20,22,23,31]$.

Currently there are several different definitions of fractional integrals and derivatives, from the most famous of which are the Riemann-Liouville and Caputo fractional derivatives to other less well known definitions. A generalization of the derivatives of both Riemann-Liouville and Caputo was given by R. Hilfer in [11], known as the fractional Hilfer derivative of order $\alpha$ and type $\beta \in[0,1]$. Some properties and applications of the Helfer derivative are given in $[12,13]$ and the references mentioned therein. Prime value problems involving fractional Hilfer derivatives have been studied by several authors, see $[9,10,26]$. However, in the literature there are few papers on the boundary

Received: 8 Oct 2021.

Key words and phrases. $\varphi$-Hilfer derivative; existence of solution; fixed point; Hyers-Ulam stability. 
value problems of the fractional Hilfer derivatives. The authors set out in [2] non-local value problems for derivatives of Helfer's fractions. For some recent work on boundary value problems with fractional Hilfer derivatives, we refer to the papers in [28-30].

Some authors have worked on the EU of solutions for fractional DEs with $p$-Laplacian operator. We cite, for example; Li., Wang., Khan et al. $[15,19,27]$ studieds a nonlinear fractional DE with p-Laplacian operator for the EU of solutions.

H. Khan, T. Abdeljawad, M. Aslam, R. A. Khan and A. Khan [16]. worked on the following proposal for the existence of a positive solution (EPS) and stability analysis:

$$
\left\{\begin{array}{c}
\mathcal{D}^{r_{1}} \psi_{p}\left[\mathcal{D}^{r_{2}}\left(u(t)-v_{1}(t, u(t))\right)\right]=-A(t) v_{2}(t, u(t-\tau)) \\
\left.\psi_{p}\left[\mathcal{D}^{r_{2}}\left(u(t)-v_{1}(t, u(t))\right)\right]\right|_{t=0}=\left.\psi_{p}\left[\mathcal{D}^{r_{2}}\left(u(t)-v_{1}(t, u(t))\right)^{\prime}\right]\right|_{t=0}=0 \\
u(0)=u(1)=0 \\
{\left.\left[\mathcal{I}^{2-r_{2}}\left(u(t)-v_{1}(t, u(t))\right)\right]\right|_{t=0}=0}
\end{array}\right.
$$

where $0<r_{1}<1<r_{2}<2$, and $v_{1}, v_{2}$ are continuous but singular at some points. The fractional derivatives $\mathcal{D}^{r_{1}}$ and $\mathcal{D}^{r_{2}}$ are taken in the Caputo sense and in the Riemann-Liouville sense, respectively, and $\psi_{p}(z)=|z|^{p-2} z$ denotes the $p$-Laplacian operator and satisfies $\frac{1}{p}+\frac{1}{q}=$ $1,\left(\psi_{p}\right)^{-1}=\psi_{q}$

A. Devi, A. Kumar, D. Baleanu and A. Khan [7]. worked on the EU and HU stability results, for nonliner FDEs involving Caputo fractional derivatives of distinct orders with $\psi_{p}$ Laplacian operator:

$$
\left\{\begin{array}{c}
{ }^{c} \mathcal{D}^{r_{1}} \psi_{p}\left[{ }^{c} \mathcal{D}^{r_{2}}\left(u(t)-\sum_{i=1}^{m} v_{i}(t)\right)\right]=-w(t, u(t)), t \in(0,1] \\
\left.\psi_{p}\left[{ }^{c} \mathcal{D}^{r_{2}}\left(u(t)-\sum_{i=1}^{m} v_{i}(t)\right)\right]\right|_{t=0}=0, \\
u(0)=\sum_{i=1}^{m} v_{i}(0), \\
u^{\prime}(1)=\sum_{i=1}^{m} v_{i}^{\prime}(1), \\
u^{j}(0)=\sum_{i=1}^{m} v_{i}^{j}(0), \text { for } j=2,3, \ldots, n-1,
\end{array}\right.
$$

where $0<r_{1} \leq 1, n-1<r_{2} \leq n, n \geq 4$, and $v_{i}, w$ are continuous functions. ${ }^{c} \mathcal{D}^{r_{1}}$ and ${ }^{c} \mathcal{D}^{r_{2}}$ denotes the derivative of fractional order $r_{1}$ and $r_{2}$ in Caputo's sense, respectively, and $\psi_{p}(z)=|z|^{p-2} z$ denotes the $p$-Laplacian operator and satisfies $\frac{1}{p}+\frac{1}{q}=1,\left(\psi_{p}\right)^{-1}=\psi_{q}$.

In the present research work, we study the existence and uniqueness of a solution (EPS) and stability analysis which includes the $\varphi$-Hilfer fractional-order of the form: 


$$
\left\{\begin{array}{c}
{ }^{H} \mathcal{D}_{a^{+}}^{\alpha_{1}, \beta_{1} ; \varphi} \psi_{p}\left({ }^{H} \mathcal{D}_{a^{+}}^{\alpha_{2}, \beta_{2} ; \varphi} u\right)(t)=h\left(t, u(t),{ }^{R L} \mathcal{D}_{a^{+}}^{\mu ; \varphi} u(t)\right), \quad t \in J=(a, b] \\
u(a)=0, u(b)=\sum_{i=1}^{n} \lambda_{i} u\left(\zeta_{i}\right), \\
\psi_{p}\left({ }^{H} \mathcal{D}_{a^{+}}^{\alpha_{2}, \beta_{2} ; \varphi} u\right)(a)=0, \\
\text { and } \psi_{p}\left({ }^{H} \mathcal{D}_{a^{+}}^{\alpha_{2}, \beta_{2} ; \varphi} u(b)\right)=\mathcal{I}_{a^{+}}^{\rho ; \varphi} u(\zeta), a<\zeta_{i}<b,
\end{array}\right.
$$

Here, we take ${ }^{H} \mathcal{D}_{0^{+}}^{\alpha_{1}, \beta ; \varphi},{ }^{H} \mathcal{D}_{0^{+}}^{\alpha_{2}, \beta ; \varphi}$, are the $\varphi$-Hilfer fractional derivative of orders $\alpha_{1}, \alpha_{2}, 1<$ $\alpha_{1}, \alpha_{2}<2$ and $\beta_{1}, \beta_{2}$ two parameters $0 \leq \beta_{1}, \beta_{2} \leq 1,{ }^{R L} \mathcal{D}_{a^{+}}^{\kappa ; \varphi}$ the $\varphi$-Riemann-Liouville fractional derivative of order $\mu$ where $\mu<\alpha_{2}$, and $\mathcal{I}_{0^{+}}^{\rho ; \varphi}$ the left-sided $\varphi$-Riemann Liouville fractional integral of order $\rho$, where $\rho>0$, and $\psi_{p}(z)=|z|^{p-2} z$ denotes the $p$-Laplacian operator and satisfies $\frac{1}{p}+\frac{1}{q}=1,\left(\psi_{p}\right)^{-1}=\psi_{q}$, and $\varphi: J \rightarrow \mathbb{R}$ be an increasing function such that $\varphi^{\prime}(t) \neq 0$, for all $t \in J$, and $f: J \times \mathbb{R} \times \mathbb{R} \rightarrow \mathbb{R}$, is given function will be "well defined" later.

\section{Phi-Hilfer Derivatives Calculus}

In this section, we introduce some notations and definitions of Phi-Hilfer Derivatives Calculus and present preliminary results needed in our proofs later, for details, see $[17,24,25]$.

Let $\varphi:[a, b] \rightarrow \mathbb{R}$ be an increasing function with $\varphi^{\prime}(t) \neq 0$, for all $t \in J$, and let $C([a, b], \mathbb{R})$ be the Banach space.

For all $v>-1$ and $s, t \in[0, \infty),(t \geq s)$, we pose $\varphi_{v}(t, s)=(\varphi(t)-\varphi(s))^{v}$.

Definition 1. Let $(a, b),(-\infty \leq a<b \leq \infty)$ be a finite or infinite interval of the half-axis $(0, \infty)$ and $\alpha>0$. In addition, let $\varphi(t)$ be a positive increasing function on $(a, b]$, which has a continuous derivative $\varphi^{\prime}(t)$ on $(a, b)$. The $\varphi$-Riemann-Liouville fractional integral of a function $u$ with respect to another function $\varphi$ on $[a, b]$ is defined by

$$
\mathcal{I}_{a^{+}}^{\alpha ; \varphi} u(t)=\frac{1}{\Gamma(\alpha)} \int_{a}^{t} \varphi^{\prime}(s) \varphi_{\alpha-1}(t, s) u(s) d s,
$$

where $\Gamma($.$) is the Gamma function.$

Definition 2. Let $n \in \mathbb{N}$ and let $\varphi, u \in C^{n}(J)$ be two functions such that $\varphi$ is increasing and $\varphi^{\prime}(t) \neq 0$, for all $t \in(a, b]$. The left-sided $\varphi$-Riemann Liouville fractional derivative of a function $u$ of order $\alpha$ is defined by

$$
\begin{aligned}
\mathcal{D}_{a^{+}}^{\alpha ; \varphi} u(t) & =\left(\frac{1}{\varphi^{\prime}(t)} \frac{d}{d t}\right)^{n} \mathcal{I}_{a^{+}}^{n-\alpha ; \varphi} u(t) \\
& =\frac{1}{\Gamma(n-\alpha)}\left(\frac{1}{\varphi^{\prime}(t)} \frac{d}{d t}\right)^{n} \int_{a}^{t} \varphi^{\prime}(s) \varphi_{n-\alpha-1}(t, s) u(s) d s
\end{aligned}
$$


where $n=[\alpha]+1,[\alpha]$ represents the integer part of the real number $\alpha$.

Definition 3. Let $n-1<\alpha<n$ with $n \in \mathbb{N},[a, b]$ is the interval such that $-\infty \leq a<b \leq \infty$ and $\varphi, u \in C^{n}([a, b], \mathbb{R})$ two functions such that $\varphi$ is increasing and $\varphi^{\prime}(t) \neq 0$, for all $t \in[a, b]$. The $\varphi$-Hilfer fractional derivative of a function $u$ of order a and type $0 \leq \beta \leq 1$ is defined by

$$
{ }^{H} \mathcal{D}_{a^{+}}^{\alpha, \beta ; \varphi} u(t)=\mathcal{I}_{a^{+}}^{\beta(n-\alpha) ; \varphi}\left(\frac{1}{\varphi^{\prime}(t)} \frac{d}{d t}\right)^{n} \mathcal{I}_{a^{+}}^{(1-\beta)(n-\alpha) ; \varphi} u(t)=\mathcal{I}_{a^{+}}^{\gamma-\alpha ; \varphi} \mathcal{D}_{a^{+}}^{\gamma ; \varphi} u(t),
$$

where $n=[\alpha]+1, \gamma-\alpha=\beta(n-\alpha)$.

\subsection{Auxiliary Lemma.}

Lemma 1. Let $\alpha, \rho>0$. Then, we have the following semigroup property given by

$$
\mathcal{I}_{a^{+}}^{\alpha ; \varphi} \mathcal{I}_{a^{+}}^{\rho ; \varphi} u(t)=\mathcal{I}_{a^{+}}^{\alpha+\rho ; \varphi} u(t), \quad t>a
$$

Next, we present the $\varphi$-fractional integral and derivatives of a power function.

Proposition 1. Let $\alpha \geq 0, \sigma>0$ and $t>a$. Then, $\varphi$-fractional integral and derivative of a power function are given by

(1) $\mathcal{I}_{a^{+}}^{\alpha, \varphi} \varphi_{\sigma-1}(t, a)(t)=\frac{\Gamma(\sigma)}{\Gamma(\alpha+\sigma)} \varphi_{\sigma+\alpha-1}(t, a)$.

(2) ${ }^{H} \mathcal{D}_{a^{+}}^{\alpha, \beta ; \varphi} \varphi_{\sigma-1}(t, a)(t)=\frac{\Gamma(\sigma)}{\Gamma(\sigma-\alpha)} \varphi_{\sigma-\alpha-1}(t, a), n-1<\alpha<n, \sigma>n$.

Lemma 2. If $u \in C^{n}([a, b], \mathbb{R}), n-1<\alpha<n, 0 \leq \beta \leq 1$ and $\gamma=\alpha+\beta(n-\alpha)$. Then

$$
\mathcal{I}_{a^{+}}^{\alpha, \varphi}\left({ }^{H} \mathcal{D}_{a^{+}}^{\alpha, \beta ; \varphi} u\right)(t)=u(t)-\sum_{k=1}^{k=n} \frac{\varphi_{\gamma-k}(t, s)}{\Gamma(\gamma-k+1)} \nabla_{\varphi}^{[n-k]} \mathcal{I}_{a^{+}}^{(1-\beta)(n-\alpha) ; \varphi} u(a), \quad t \in[a, b],
$$

where $\nabla_{\varphi}^{[n]} u(t):=\left(\frac{1}{\psi^{\prime}(t)} \frac{d}{d t}\right)^{n} u(t)$.

Lemma 3. Let $u \in C^{n}[a, b]$ and $0<q<1$, we have

$$
\left|\mathcal{I}_{a^{+}}^{q ; \varphi} u\left(t_{2}\right)-\mathcal{I}_{a^{+}}^{q ; \varphi} u\left(t_{1}\right)\right| \leq \frac{2\|u\|}{\Gamma(q+1)} \varphi_{q}\left(t_{2}, t_{1}\right) .
$$

Lemma 4. ([14]) For the $p$-Laplacian operator $\psi_{p}$, the following conditions hold true:

(1) If $\left|\delta_{1}\right|,\left|\delta_{2}\right| \geq \rho>0,1<p \leq 2, \delta_{1} \delta_{2}>0$, then

$$
\left|\psi_{p}\left(\delta_{1}\right)-\psi_{p}\left(\delta_{2}\right)\right| \leq(p-1) \rho^{p-2}\left|\delta_{1}-\delta_{2}\right| \text {. }
$$

(2) If $p>2,\left|\delta_{1}\right|,\left|\delta_{2}\right| \leq \rho_{*}>0$, then

$$
\left|\psi_{p}\left(\delta_{1}\right)-\psi_{p}\left(\delta_{2}\right)\right| \leq(p-1) \rho_{*}^{p-2}\left|\delta_{1}-\delta_{2}\right| .
$$

Lemma 5. [9] For nonnegative $a_{i}, i=1, \ldots, k$,

$$
\left(\sum_{i=1}^{k} a_{i}\right)^{q} \leq k^{q-1}\left(\sum_{i=1}^{k} a_{i}^{q}\right), q \geq 1 .
$$


Lemma 6. Let $a \geq 0,1<\alpha_{1}, \alpha_{2}<2,0 \leq \beta_{1}, \beta_{2} \leq 1$, and $2-\gamma_{1}=\left(1-\beta_{1}\right)\left(2-\alpha_{1}\right), 2-\gamma_{2}=$ $\left(1-\beta_{2}\right)\left(2-\alpha_{2}\right)$. For $f \in C(J,, \mathbb{R}, \mathbb{R})$, the unique solution of the sequential Hilfer fractional boundary value problem

$$
\begin{gathered}
{ }^{H} \mathcal{D}_{a^{+}}^{\alpha_{1}, \beta_{1} ; \varphi} \psi_{p}\left({ }^{H} \mathcal{D}_{a^{+}}^{\alpha_{2}, \beta_{2} ; \varphi} u\right)(t)=f(t), \quad t \in J=[a, b], \\
u(a)=0, u(b)=\sum_{i=1}^{n} \lambda_{i} u\left(\zeta_{i}\right), \\
\psi_{p}\left({ }^{H} \mathcal{D}_{a^{+}}^{\alpha_{2}, \beta_{2} ; \varphi} u\right)(a)=0, \\
\text { and } \psi_{p}\left({ }^{H} \mathcal{D}_{a^{+}}^{\alpha_{2}, \beta_{2} ; \varphi} u(b)\right)=\mathcal{I}_{a^{+}}^{\rho ; \varphi} u(\zeta), a<\zeta, \zeta_{i}<b,
\end{gathered}
$$

is given by

$$
\begin{aligned}
u(t)= & \frac{1}{\Gamma\left(\alpha_{2}\right)} \int_{a}^{t} \varphi^{\prime}(s) \varphi_{\alpha_{2}-1}(t, s) X(s, a) d s \\
& -\frac{\varphi_{\gamma_{2}-1}(t, a)}{\Gamma\left(\alpha_{2}\right) \varphi_{\gamma_{2}-1}(b, a)} \int_{a}^{b} \varphi^{\prime}(t) \varphi_{\alpha_{2}-1}(b, t) X(t, a) d t \\
& +\frac{\varphi_{\gamma_{2}-1}(t, a)}{\varphi_{\gamma_{2}-1}(b, a)} \sum_{i=1}^{n} \lambda_{i} u\left(\zeta_{i}\right) .
\end{aligned}
$$

where

$$
\begin{aligned}
X(s, a) & =\psi_{q}\left(\frac{1}{\Gamma\left(\alpha_{1}\right)} \int_{a}^{s} \varphi^{\prime}(s) \varphi_{\alpha_{1}-1}(s, z) f(z) d z+\frac{\left(\mathcal{I}_{0^{+}}^{\rho ; \varphi} u(\zeta)-\mathcal{I}_{0^{+}}^{\alpha_{1} ; \varphi} f(b)\right)}{\varphi_{\gamma_{1}-1}(b, a)} \varphi_{\gamma_{1}-1}(s, a)\right) \\
\mathcal{I}_{0^{+}}^{\rho ; \varphi} u(\zeta) & =\frac{1}{\Gamma(\rho)} \int_{a}^{\zeta} \varphi^{\prime}(s) \varphi_{\rho}(\zeta, s) u(s) d s, \\
\mathcal{I}_{0^{+}}^{\alpha_{1} ; \varphi} f(b) & =\frac{1}{\Gamma\left(\alpha_{1}\right)} \int_{a}^{b} \varphi^{\prime}(s) \varphi_{\alpha_{1}-1}(b, s) f(s) d s .
\end{aligned}
$$

Proof. Assume that $u$ is a solution of the sequential nonlocal boundary value Problems (3.6) and (2.3). Applying the two operators $\mathcal{I}_{a^{+}}^{\alpha_{1} ; \varphi}, \mathcal{I}_{a^{+}}^{\alpha_{2} ; \varphi}$ to both sides of Equation (3.6) and using Lemma 2 and Proposition 1, we obtain

$$
\psi_{p}\left({ }^{H} \mathcal{D}_{a^{+}}^{\alpha_{2}, \beta_{2} ; \varphi} u\right)(t)=\mathcal{I}_{a^{+}}^{\alpha_{1} ; \varphi} f(t)+\frac{m_{0}}{\Gamma\left(\gamma_{1}-1\right)} \varphi_{\gamma_{1}-2}(t, a)+\frac{m_{1}}{\Gamma\left(\gamma_{1}\right)} \varphi_{\gamma_{1}-1}(t, a),
$$

where $m_{0}, m_{1} \in \mathbb{R}$, and $2-\gamma_{1}=\left(1-\beta_{1}\right)\left(2-\alpha_{1}\right)$. From the boundary condition $\psi_{p}\left({ }^{H} \mathcal{D}_{a^{+}}^{\alpha_{2}, \beta_{2} ; \varphi} u\right)(a)=0$, and if $t \rightarrow a$ then $\varphi_{\gamma_{1}-2}(t, a) \rightarrow \infty$, we get

$$
m_{0}=0
$$


and by $\psi_{p}\left({ }^{H} \mathcal{D}_{a^{+}}^{\alpha_{2}, \beta_{2} ; \varphi} u\right)(b)=\mathcal{I}_{a^{+}}^{\rho ; \varphi} u(\zeta)$, we obtain

$$
m_{1}=\frac{\Gamma\left(\gamma_{1}\right)}{\varphi_{\gamma_{1}-1}(b, a)}\left(\mathcal{I}_{0^{+}}^{\rho ; \varphi} u(\zeta)-\mathcal{I}_{0^{+}}^{\alpha_{1} ; \varphi} f(b)\right)
$$

So

$$
{ }^{H} \mathcal{D}_{a^{+}}^{\alpha_{2}, \beta_{2} ; \varphi} u(t)=\psi_{q}\left(\mathcal{I}_{a^{+}}^{\alpha_{1} ; \varphi} f(t)+\frac{\varphi_{\gamma_{1}-1}(t, a)}{\varphi_{\gamma_{1}-1}(b, a)}\left(\mathcal{I}_{0^{+}}^{\rho ; \varphi} u(\zeta)-\mathcal{I}_{0^{+}}^{\alpha_{1} ; \varphi} f(b)\right)\right)
$$

by (2.4)we have

$$
\begin{aligned}
u(t)= & \mathcal{I}_{a^{+}}^{\alpha_{2} ; \varphi}\left[\psi_{q}\left(\mathcal{I}_{a^{+}}^{\alpha_{1} ; \varphi} f(t)+\frac{\varphi_{\gamma_{1}-1}(t, a)}{\varphi_{\gamma_{1}-1}(b, a)}\left(\mathcal{I}_{0^{+}}^{\rho ; \varphi} u(\zeta)-\mathcal{I}_{0^{+}}^{\alpha_{1} ; \varphi} f(b)\right)\right)\right] \\
& +\frac{m_{2}}{\Gamma\left(\gamma_{2}-1\right)} \varphi_{\gamma_{2}-2}(t, a)+\frac{m_{3}}{\Gamma\left(\gamma_{2}\right)} \varphi_{\gamma_{2}-1}(t, a),
\end{aligned}
$$

where $m_{2}, m_{3} \in \mathbb{R}$, and $2-\left(1-\beta_{2}\right)\left(2-\alpha_{2}\right)=\gamma_{2}$.and if $t \rightarrow a$ then $\varphi_{\gamma_{2}-2}(t, a) \rightarrow \infty$, we get

By conditions $u(a)=0$, and $\lim _{t \rightarrow 0} t^{\gamma_{2}-2}=\infty$, we get

$$
m_{2}=0
$$

So

$$
u(t)=\mathcal{I}_{a^{+}}^{\alpha_{2} ; \varphi}\left[\psi_{q}\left(\mathcal{I}_{a^{+}}^{\alpha_{1} ; \varphi} f(t)+\frac{\varphi_{\gamma_{1}-1}(t, a)}{\varphi_{\gamma_{1}-1}(b, a)}\left(\mathcal{I}_{0^{+}}^{\rho ; \varphi} u(\zeta)-\mathcal{I}_{0^{+}}^{\alpha_{1} ; \varphi} f(b)\right)\right)\right]+\frac{m_{3}}{\Gamma\left(\gamma_{2}\right)} \varphi_{\gamma_{2}-1}(t, a)
$$

By conditions $u(b)=\sum_{i=1}^{n} \lambda_{i} u\left(\zeta_{i}\right)$, we get

$$
\begin{aligned}
m_{3}= & \frac{\Gamma\left(\gamma_{2}\right)}{\varphi_{\gamma_{2}-1}(b, a)} \sum_{i=1}^{n} \lambda_{i} u\left(\zeta_{i}\right) \\
& -\frac{\Gamma\left(\gamma_{2}\right)}{\varphi_{\gamma_{2}-1}(b, a)} \mathcal{I}_{a^{+}}^{\alpha_{2} ; \varphi}\left[\psi_{q}\left(\mathcal{I}_{a^{+}}^{\alpha_{1} ; \varphi} f(t)+\frac{\varphi_{\gamma_{1}-1}(t, a)}{\varphi_{\gamma_{1}-1}(b, a)}\left(\mathcal{I}_{0^{+}}^{\rho ; \varphi} u(\zeta)-\mathcal{I}_{0^{+}}^{\alpha_{1} ; \varphi} f(b)\right)\right)\right]_{t=b} .
\end{aligned}
$$

Then

$$
\begin{aligned}
u(t)= & \mathcal{I}_{a^{+}}^{\alpha_{2} ; \varphi}\left[\psi_{q}\left(\mathcal{I}_{a^{+}}^{\alpha_{1} ; \varphi} f(t)+\frac{\varphi_{\gamma_{1}-1}(t, a)}{\varphi_{\gamma_{1}-1}(b, a)}\left(\mathcal{I}_{0^{+}}^{\rho ; \varphi} u(\zeta)-\mathcal{I}_{0^{+}}^{\alpha_{1} ; \varphi} f(b)\right)\right)\right]+\frac{\varphi_{\gamma_{2}-1}(t, a)}{\varphi_{\gamma_{2}-1}(b, a)} \sum_{i=1}^{n} \lambda_{i} u\left(\zeta_{i}\right) \\
& -\frac{\varphi_{\gamma_{2}-1}(t, a)}{\varphi_{\gamma_{2}-1}(b, a)} \mathcal{I}_{a^{+}}^{\alpha_{2} ; \varphi}\left[\psi_{q}\left(\mathcal{I}_{a^{+}}^{\alpha_{1} ; \varphi} f(t)+\frac{\varphi_{\gamma_{1}-1}(t, a)}{\varphi_{\gamma_{1}-1}(b, a)}\left(\mathcal{I}_{0^{+}}^{\rho ; \varphi} u(\zeta)-\mathcal{I}_{0^{+}}^{\alpha_{1} ; \varphi} f(b)\right)\right)\right]_{t=b} .
\end{aligned}
$$

This finishes the proof. 


\section{Conjecture 1.}

$$
\begin{aligned}
& R L \mathcal{D}_{a^{+}}^{\mu ; \varphi} u(t)=\frac{1}{\Gamma\left(\alpha_{2}-\mu\right)} \int_{a}^{t} \varphi^{\prime}(s) \varphi_{\alpha_{2}-\mu-1}(t, s) X(s, a) d s \\
& +\frac{\Gamma\left(\gamma_{2}\right)}{\Gamma\left(\gamma_{2}-\mu\right)} \frac{\varphi_{\gamma_{2}-\mu-1}(t, a)}{\varphi_{\gamma_{2}-1}(b, a)} \sum_{i=1}^{n} \lambda_{i} u\left(\zeta_{i}\right) \\
& -\frac{\Gamma\left(\gamma_{2}\right)}{\Gamma\left(\alpha_{2}\right) \Gamma\left(\gamma_{2}-\mu\right)} \frac{\varphi_{\gamma_{2}-\mu-1}(t, a)}{\varphi_{\gamma_{2}-1}(b, a)} \int_{a}^{b} \varphi^{\prime}(t) \varphi_{\alpha_{2}-1}(b, t) X(t, a) d t .
\end{aligned}
$$

\section{Main Results}

In this section, we present to the reader our main results on the existence and stability for the above problem. We begin by considering the space

$$
C_{\varphi}^{\mu}=\left\{u: u,{ }^{R L} \mathcal{D}_{a^{+}}^{\mu ; \varphi} u \in C([a, b], \mathbb{R})\right\}
$$

with the norm

$$
\|u\|_{C_{\varphi}^{\mu}}=\|u\|_{C}+\left\|{ }^{R L} \mathcal{D}_{a^{+}}^{\mu ; \varphi} u\right\|_{C}
$$

such that

$$
\|u\|_{C}=\sup _{t \in[a, b]}|u(t)|, \text { and }\left\|{ }^{R L} \mathcal{D}_{a^{+}}^{\mu ; \varphi} u\right\|_{C}=\sup _{t \in[a, b]}\left|R L \mathcal{D}_{a^{+}}^{\mu ; \varphi} u(t)\right|
$$

3.1. Criteria For Uniqueness Solution. Now, wee need to consider the following assumptions:

$\left.\mathcal{H}_{1}\right) h$ is continuous function.

$\mathcal{H}_{2}$ ) There exists a constant $\Upsilon>0$, such that

$$
|h(t, u, v)-h(t, x, y)| \leq \Upsilon(|u-x|+|v-y|)
$$

with $t \in[a, b],(u, v, x, y) \in \mathbb{R}^{4}$.

$\mathcal{H}_{3}$ ) There exists two continuous functions $\pi_{1}, \pi_{2}:[a, b] \rightarrow \mathbb{R}^{+}$, such that

$$
|h(t, u, v)| \leq \pi_{1}(t)|u(t)|+\pi_{2}(t)|v(t)|
$$

where

$$
\pi_{1}^{*}=\sup _{t \in[a, b]}\left|\pi_{1}(t)\right|, \quad \text { and } \pi_{2}^{*}=\sup _{t \in[a, b]}\left|\pi_{2}(t)\right|
$$

Now, we define the following quantities: 


$$
\begin{aligned}
\varphi_{q}(b, a) & =M^{q}, \\
\Omega & =3^{q-2}\left[\left(\frac{2 M^{\alpha_{1}}}{\Gamma\left(\alpha_{1}+1\right)}\right)^{q-1}\left(\left(\pi_{1}^{*}\right)^{q-1}+\left(\pi_{2}^{*}\right)^{q-1}\right)+\left(\frac{M^{\rho}}{\Gamma(\rho+1)}\right)^{q-1}\right] \\
\Lambda_{1} & =\frac{2 \cdot \Omega \cdot M^{\alpha_{2}}}{\Gamma\left(\alpha_{2}+1\right)}, \\
\Lambda_{2} & =\left(\sum_{i=1}^{n}\left|\lambda_{i}\right|\right), \\
\Lambda_{3} & =\frac{\Omega \cdot M^{\alpha_{2}-\mu}}{\Gamma\left(\alpha_{2}-\mu+1\right)}+\frac{\Omega \cdot \Gamma\left(\gamma_{2}\right) M^{\alpha_{2}-\mu}}{\Gamma\left(\alpha_{2}+1\right) \Gamma\left(\gamma_{2}-\mu\right)}, \\
\Lambda_{4} & =\frac{\Gamma\left(\gamma_{2}\right) M^{-\mu}}{\Gamma\left(\gamma_{2}-\mu\right)} \Lambda_{2} .
\end{aligned}
$$

Based on the above hypotheses, we present to the reader the following result.

Theorem 1. Under $\mathcal{H}_{2}$ and $\mathcal{H}_{3}$ the equation (1.1) has a solution.

Proof. Firstly: We begin this proof by defining the operator $\mathbb{G}: C_{\varphi}^{\mu} \rightarrow C_{\varphi}^{\mu}$ by:

$$
\begin{aligned}
(\mathbb{G} u)(t)= & \frac{1}{\Gamma\left(\alpha_{2}\right)} \int_{a}^{t} \varphi^{\prime}(s) \varphi_{\alpha_{2}-1}(t, s) X_{u}(s, a) d s-\frac{\varphi_{\gamma_{2}-1}(t, a)}{\Gamma\left(\alpha_{2}\right) \varphi_{\gamma_{2}-1}(b, a)} \int_{a}^{b} \varphi^{\prime}(t) \varphi_{\alpha_{2}-1}(b, t) X_{u}(t, a) d t \\
& +\frac{\varphi_{\gamma_{2}-1}(t, a)}{\varphi_{\gamma_{2}-1}(b, a)} \sum_{i=1}^{n} \lambda_{i} u\left(\zeta_{i}\right) .
\end{aligned}
$$

where

$$
X_{u}(s, a)=\psi_{q}\left(\frac{1}{\Gamma\left(\alpha_{1}\right)} \int_{a}^{s} \varphi^{\prime}(s) \varphi_{\alpha_{1}-1}(s, z) h_{u}(z) d z+\frac{\left(\mathcal{I}_{0^{+}}^{\rho ; \varphi} u(\zeta)-\mathcal{I}_{0^{+}}^{\alpha_{1} ; \varphi} h_{u}(b)\right)}{\varphi_{\gamma_{1}-1}(b, a)} \varphi_{\gamma_{1}-1}(s, a)\right)
$$

where

$$
h_{u}(t)=h\left(t, u(t),{ }^{R L} \mathcal{D}_{a^{+}}^{\mu ; \varphi} u(t)\right) .
$$

We consider the set $\mathcal{U}_{r}=\left\{u \in C_{\varphi}^{\mu}:\|u\|_{C_{\varphi}^{\mu}} \leq r\right\}$, so that

$$
\max \left\{\left(2\left(\Lambda_{1}+\Lambda_{3}\right)\right)^{\frac{1}{2-q}}, 2\left(\Lambda_{2}+\Lambda_{4}\right)\right\} \leq r
$$


We show that $\mathbb{G} \mathcal{U}_{r} \subset \mathcal{U}_{r}$. For any $u \in \mathcal{U}_{r}$, and by Lemma 5 we have

$$
\begin{aligned}
& \left|X_{u}(s, a)\right| \\
= & \left|\left[\psi_{q}\left(\frac{1}{\Gamma\left(\alpha_{1}\right)} \int_{a}^{s} \varphi^{\prime}(s) \varphi_{\alpha_{1}-1}(s, z) h_{u}(z) d z+\frac{\left(\mathcal{I}_{0^{+}}^{\rho ; \varphi} u(\zeta)-\mathcal{I}_{0^{+}}^{\alpha_{1} ; \varphi} h_{u}(b)\right)}{\varphi_{\gamma_{1}-1}(b, a)} \varphi_{\gamma_{1}-1}(s, a)\right)\right]\right| \\
\leq & \sup _{t \in[a, b]}\left|\frac{1}{\Gamma\left(\alpha_{1}\right)} \int_{a}^{s} \varphi^{\prime}(s) \varphi_{\alpha_{1}-1}(s, z) h_{u}(z) d z+\mathcal{I}_{0^{+}}^{\rho ; \varphi} u(\zeta)+\mathcal{I}_{0^{+}}^{\alpha_{1} ; \varphi} h_{u}(b)\right|^{q-1} \\
\leq & 3^{q-2} \sup _{t \in[a, b]}\left(\left(\frac{1}{\Gamma\left(\alpha_{1}\right)} \int_{a}^{s} \varphi^{\prime}(s) \varphi_{\alpha_{1}-1}(s, z) h_{u}(z) d z\right)^{q-1}+\left(\mathcal{I}_{0^{+}}^{\rho ; \varphi} u(\zeta)\right)^{q-1}+\left(\mathcal{I}_{0^{+}}^{\alpha_{1} ; \varphi} h_{u}(b)\right)^{q-1}\right) \\
\leq & 3^{q-2}\left[2\left(\frac{\pi_{1}^{*} M^{\alpha_{1}}}{\Gamma\left(\alpha_{1}+1\right)}\|u\|_{C}+\frac{\pi_{2}^{*} M^{\alpha_{1}}}{\Gamma\left(\alpha_{1}+1\right)}\|\|^{R L} \mathcal{D}_{a^{+}}^{\mu ; \varphi} u \|_{C}\right)^{q-1}+\left(\frac{M^{\rho}}{\Gamma(\rho+1)}\right)^{q-1}\left(\|u\|_{C}\right)^{q-1}\right] \\
\leq & 3^{q-2}\left[\left(\frac{2 . \pi_{1}^{*} M^{\alpha_{1}}}{\Gamma\left(\alpha_{1}+1\right)}\right)^{q-1}\left(\|u\|_{C}\right)^{q-1}+\left(\frac{2 . \pi_{2}^{*} M^{\alpha_{1}}}{\Gamma\left(\alpha_{1}+1\right)}\right)^{q-1}\left(\left\|\mathcal{D}^{R L} \mathcal{D}_{a^{+}}^{\mu ; \varphi} u\right\|_{C}\right)^{q-1}+\left(\frac{M^{\rho}}{\Gamma(\rho+1)}\right)^{q-1}\left(\|u\|_{C}\right)^{q-1}\right] \\
\leq & 3^{q-2}\left[\left(\frac{2 M^{\alpha_{1}}}{\Gamma\left(\alpha_{1}+1\right)}\right)^{q-1}\left(\left(\pi_{1}^{*}\right)^{q-1}+\left(\pi_{2}^{*}\right)^{q-1}\right)+\left(\frac{M^{\rho}}{\Gamma(\rho+1)}\right)^{q-1}\right] r^{q-1} \\
\leq & \Omega^{q . r^{q-1}} .
\end{aligned}
$$

Then

$$
\begin{aligned}
& \sup _{t \in[a, b]}|(\mathbb{G} u)(t)| \\
\leq & \sup _{t \in[a, b]} \mid \frac{1}{\Gamma\left(\alpha_{2}\right)} \int_{a}^{t} \varphi^{\prime}(s) \varphi_{\alpha_{2}-1}(t, s) X_{u}(s, a) d s+\frac{\varphi_{\gamma_{2}-1}(t, a)}{\Gamma\left(\alpha_{2}\right) \varphi_{\gamma_{2}-1}(b, a)} \int_{a}^{b} \varphi^{\prime}(t) \varphi_{\alpha_{2}-1}(b, t) X_{u}(t, a) d t \\
& +\frac{\varphi_{\gamma_{2}-1}(t, a)}{\varphi_{\gamma_{2}-1}(b, a)} \sum_{i=1}^{n} \lambda_{i} u\left(\zeta_{i}\right) \mid \\
\leq & \frac{2 M^{\alpha_{2}}}{\Gamma\left(\alpha_{2}+1\right)}\left|X_{u}\right|+\left(\sum_{i=1}^{n}\left|\lambda_{i}\right|\right) \sup _{t \in[a, b]}|u(t)| \\
\leq & \frac{2 . \Omega \cdot M^{\alpha_{2}}}{\Gamma\left(\alpha_{2}+1\right)} r^{q}+\left(\sum_{i=1}^{n}\left|\lambda_{i}\right|\right) r . \\
\leq & \Lambda_{1} r^{q}+\Lambda_{2} r .
\end{aligned}
$$

Also, we have

$$
\begin{aligned}
& \sup _{t \in[a, b]}\left|\left(R L \mathcal{D}_{a^{+}}^{\mu ; \varphi} \mathbb{G} u\right)(t)\right| \\
\leq & \sup _{t \in[a, b]} \mid \frac{1}{\Gamma\left(\alpha_{2}-\mu\right)} \int_{a}^{t} \varphi^{\prime}(s) \varphi_{\alpha_{2}-\mu-1}(t, s) X_{u}(s, a) d s+\frac{\Gamma\left(\gamma_{2}\right)}{\Gamma\left(\gamma_{2}-\mu\right)} \frac{\varphi_{\gamma_{2}-\mu-1}(t, a)}{\varphi_{\gamma_{2}-1}(b, a)} \sum_{i=1}^{n} \lambda_{i} u\left(\zeta_{i}\right)
\end{aligned}
$$




$$
\begin{aligned}
& -\frac{\Gamma\left(\gamma_{2}\right)}{\Gamma\left(\alpha_{2}\right) \Gamma\left(\gamma_{2}-\mu\right)} \frac{\varphi_{\gamma_{2}-\mu-1}(t, a)}{\varphi_{\gamma_{2}-1}(b, a)} \int_{a}^{b} \varphi^{\prime}(t) \varphi_{\alpha_{2}-1}(b, t) X_{u}(t, a) d t \mid \\
\leq & {\left[\frac{M^{\alpha_{2}-\mu}}{\Gamma\left(\alpha_{2}-\mu+1\right)}+\frac{\Gamma\left(\gamma_{2}\right) M^{\alpha_{2}-\mu}}{\Gamma\left(\alpha_{2}+1\right) \Gamma\left(\gamma_{2}-\mu\right)}\right]\left|X_{u}\right|+\frac{\Gamma\left(\gamma_{2}\right) M^{-\mu}}{\Gamma\left(\gamma_{2}-\mu\right)}\left(\sum_{i=1}^{n}\left|\lambda_{i}\right|\right) \sup _{t \in[a, b]}|u(t)| } \\
\leq & {\left[\frac{\Omega \cdot M^{\alpha_{2}-\mu}}{\Gamma\left(\alpha_{2}-\mu+1\right)}+\frac{\Omega \cdot \Gamma\left(\gamma_{2}\right) M^{\alpha_{2}-\mu}}{\Gamma\left(\alpha_{2}+1\right) \Gamma\left(\gamma_{2}-\mu\right)}\right] r^{q-1}+\frac{\Gamma\left(\gamma_{2}\right) M^{-\mu}}{\Gamma\left(\gamma_{2}-\mu\right)}\left(\sum_{i=1}^{n}\left|\lambda_{i}\right|\right) r } \\
\leq & \Lambda_{3} r^{q-1}+\Lambda_{4} r .
\end{aligned}
$$

By (3.1) and (3.2), we find

$$
\begin{aligned}
\|u\|_{C_{\varphi}^{\mu}} & =\sup _{t \in[a, b]}|(\mathbb{G} u)(t)|_{C}+\sup _{t \in[a, b]}\left|\left({ }^{R L} \mathcal{D}_{a^{+}}^{\mu ; \varphi} \mathbb{G} u\right)(t)\right|_{C} \\
& \leq\left(\Lambda_{1}+\Lambda_{3}\right) r^{q-1}+\left(\Lambda_{2}+\Lambda_{4}\right) r \\
& \leq r .
\end{aligned}
$$

that is $\mathbb{G} \mathcal{U}_{r}$ belongs to $\mathcal{U}_{r}$ on $[a, b]$.

Next, we prove that $\mathbb{G}$ is completely continuous. For any $u \in \mathcal{U}_{r}$ and $t_{1}, t_{2} \in[a ; b]$ such that $t_{1}<t_{2}$, by Lemma 3, we have

$$
\begin{aligned}
& \sup _{t \in[a, b]}\left|(\mathbb{G} u)\left(t_{2}\right)-(\mathbb{G} u)\left(t_{1}\right)\right| \\
\leq & \sup _{t \in[a, b]} \mid \frac{1}{\Gamma\left(\alpha_{2}\right)} \int_{a}^{t_{2}} \varphi^{\prime}(s) \varphi_{\alpha_{2}-1}\left(t_{2}, s\right) X_{u}(s, a) d s-\frac{1}{\Gamma\left(\alpha_{2}\right)} \int_{a}^{t_{1}} \varphi^{\prime}(s) \varphi_{\alpha_{2}-1}\left(t_{1}, s\right) X_{u}(s, a) d s \\
& +\frac{\varphi_{\gamma_{2}-1}\left(t_{2}, a\right)-\varphi_{\gamma_{2}-1}\left(t_{1}, a\right)}{\Gamma\left(\alpha_{2}\right) \varphi_{\gamma_{2}-1}(b, a)} \int_{a}^{b} \varphi^{\prime}(t) \varphi_{\alpha_{2}-1}(b, t) X_{u}(t, a) d t \\
& +\frac{\varphi_{\gamma_{2}-1}\left(t_{2}, a\right)-\varphi_{\gamma_{2}-1}\left(t_{1}, a\right)}{\varphi_{\gamma_{2}-1}(b, a)} \sum_{i=1}^{n} \lambda_{i} u\left(\zeta_{i}\right) \mid \\
\leq & \frac{\Omega \cdot r^{q-1}}{\Gamma\left(\alpha_{2}+1\right)} \varphi_{\alpha_{2}}\left(t_{2}, t_{1}\right)+\frac{\Omega \cdot M^{\alpha_{2}} \cdot r^{q-1}+\Gamma\left(\alpha_{2}+1\right) \Lambda_{2} r}{\Gamma\left(\alpha_{2}+1\right) \varphi_{\gamma_{2}-1}(b, a)} \varphi_{\gamma_{2}-1}\left(t_{2}, t_{1}\right) .
\end{aligned}
$$

Hence,

$$
\sup _{t \in[a, b]}\left|(\mathbb{G} u)\left(t_{2}\right)-(\mathbb{G} u)\left(t_{1}\right)\right| \rightarrow 0, \text { as } t_{2} \rightarrow t_{1} .
$$


Also, we can say that

$$
\begin{aligned}
& \sup _{t \in[a, b]}\left|\left({ }^{R L} \mathcal{D}_{a^{+}}^{\mu ; \varphi} \mathbb{G} u\right)\left(t_{2}\right)-\left({ }^{R L} \mathcal{D}_{a^{+}}^{\mu ; \varphi} \mathbb{G} u\right)\left(t_{1}\right)\right| \\
& \leq \sup _{t \in[a, b]} \mid \frac{1}{\Gamma\left(\alpha_{2}-\mu\right)} \int_{a}^{t_{2}} \varphi^{\prime}(s) \varphi_{\alpha_{2}-\mu-1}\left(t_{2}, s\right) X_{u}(s, a) d s-\frac{1}{\Gamma\left(\alpha_{2}-\mu\right)} \int_{a}^{t_{1}} \varphi^{\prime}(s) \varphi_{\alpha_{2}-\mu-1}\left(t_{1}, s\right) X_{u}(s, a) d s \\
& +\frac{\Gamma\left(\gamma_{2}\right)}{\Gamma\left(\gamma_{2}-\mu\right)} \frac{\varphi_{\gamma_{2}-\mu-1}\left(t_{2}, a\right)-\varphi_{\gamma_{2}-\mu-1}\left(t_{1}, a\right)}{\varphi_{\gamma_{2}-1}(b, a)} \sum_{i=1}^{n} \lambda_{i} u\left(\zeta_{i}\right) \\
& \quad+\frac{\Gamma\left(\gamma_{2}\right)}{\Gamma\left(\alpha_{2}\right) \Gamma\left(\gamma_{2}-\mu\right)} \frac{\varphi_{\gamma_{2}-\mu-1}\left(t_{2}, a\right)-\varphi_{\gamma_{2}-\mu-1}\left(t_{1}, a\right)}{\varphi_{\gamma_{2}-1}(b, a)} \int_{a}^{b} \varphi^{\prime}(t) \varphi_{\alpha_{2}-1}(b, t) X_{u}(t, a) d t \mid \\
& \leq \quad \frac{\Omega . r^{q-1}}{\Gamma\left(\alpha_{2}-\mu+1\right)} \varphi_{\alpha_{2}-\mu}\left(t_{2}, t_{1}\right) \\
& \quad+\left(\frac{\Gamma\left(\gamma_{2}\right) \Lambda_{2} r}{\Gamma\left(\gamma_{2}-\mu\right) \varphi_{\gamma_{2}-1}(b, a)}+\frac{\Gamma\left(\gamma_{2}\right) \cdot \Omega \cdot r^{q-1} \cdot M^{\alpha_{2}}}{\Gamma\left(\alpha_{2}+1\right) \Gamma\left(\gamma_{2}-\mu\right) \varphi_{\gamma_{2}-1}(b, a)}\right) \varphi_{\gamma_{2}-\mu-1}\left(t_{2}, t_{1}\right) .
\end{aligned}
$$

Hence,

$$
\sup _{t \in[a, b]}\left|\left({ }^{R L} \mathcal{D}_{a^{+}}^{\mu ; \varphi} \mathbb{G} u\right)\left(t_{2}\right)-\left({ }^{R L} \mathcal{D}_{a^{+}}^{\mu ; \varphi} \mathbb{G} u\right)\left(t_{1}\right)\right| \rightarrow 0, \text { as } t_{2} \rightarrow t_{1}
$$

As a consequence of the above three steps and thanks to Arzela-Ascoli theorem, we conclude that $\mathbb{G}$ is completely continuous.

The proof of Theorem 1 is thus completely achieved.

\subsection{Criteria For Existence of a Solution.}

Theorem 2. Assume that $\mathcal{H}_{2}$ and $\mathcal{H}_{3}$ are satisfied. Suppose that

$$
\Upsilon_{1}+\Upsilon_{2}<1
$$

where

$$
\Upsilon_{1}=\frac{2(q-1) \Delta^{q-2} M^{\alpha_{2}}}{\Gamma\left(\alpha_{2}+1\right)}\left(\frac{4 \Upsilon M^{\alpha_{1}}}{\Gamma\left(\alpha_{1}+1\right)}+\frac{M^{\rho}}{\Gamma(\rho+1)}\right)+\Lambda_{2},
$$

and

$$
\begin{aligned}
\Upsilon_{2}= & (q-1) \Delta^{q-2}\left(\frac{4 \Upsilon M^{\alpha_{1}}}{\Gamma\left(\alpha_{1}+1\right)}+\frac{M^{\rho}}{\Gamma(\rho+1)}\right)\left(\frac{M^{\alpha_{2}-\mu}}{\Gamma\left(\alpha_{2}-\mu+1\right)}+\frac{\Gamma\left(\gamma_{2}\right) M^{\alpha_{2}-\mu}}{\Gamma\left(\alpha_{2}+1\right) \Gamma\left(\gamma_{2}-\mu\right)}\right) \\
& +\frac{\Gamma\left(\gamma_{2}\right) \Lambda_{2} M^{-\mu}}{\Gamma\left(\gamma_{2}-\mu\right)} .
\end{aligned}
$$

Then, (1.1) has a uniqueness solution. 
Proof. We pass to prove that $\mathbb{G}$ is a contraction. For any $u, v \in \mathcal{U}_{r}$, we have the following estimate

$$
\begin{aligned}
& \left|X_{u}(s, a)-X_{v}(s, a)\right| \\
& =\mid \psi_{q}\left(\frac{1}{\Gamma\left(\alpha_{1}\right)} \int_{a}^{s} \varphi^{\prime}(s) \varphi_{\alpha_{1}-1}(s, z) h_{u}(z) d z+\frac{\left(\mathcal{I}_{0^{+}}^{\rho ; \varphi} u(\zeta)-\mathcal{I}_{0^{+}}^{\alpha_{1} ; \varphi} h_{u}(b)\right)}{\varphi_{\gamma_{1}-1}(b, a)} \varphi_{\gamma_{1}-1}(s, a)\right) \\
& -\psi_{q}\left(\frac{1}{\Gamma\left(\alpha_{1}\right)} \int_{a}^{s} \varphi^{\prime}(s) \varphi_{\alpha_{1}-1}(s, z) h_{v}(z) d z+\frac{\left(\mathcal{I}_{0^{+}}^{\rho ; \varphi} v(\zeta)-\mathcal{I}_{0^{+}}^{\alpha_{1} ; \varphi} h_{v}(b)\right)}{\varphi_{\gamma_{1}-1}(b, a)} \varphi_{\gamma_{1}-1}(s, a)\right) \\
& \leq(q-1) y^{q-2} \mid \frac{1}{\Gamma\left(\alpha_{1}\right)} \int_{a}^{s} \varphi^{\prime}(s) \varphi_{\alpha_{1}-1}(s, z) h_{u}(z) d z-\frac{1}{\Gamma\left(\alpha_{1}\right)} \int_{a}^{s} \varphi^{\prime}(s) \varphi_{\alpha_{1}-1}(s, z) h_{v}(z) d z \\
& +\frac{\varphi_{\gamma_{1}-1}(s, a)}{\varphi_{\gamma_{1}-1}(b, a)}\left(\mathcal{I}_{0^{+}}^{\rho ; \varphi} u(\zeta)-\mathcal{I}_{0^{+}}^{\rho ; \varphi} v(\zeta)\right)+\frac{\varphi_{\gamma_{1}-1}(s, a)}{\varphi_{\gamma_{1}-1}(b, a)}\left(\mathcal{I}_{0^{+}}^{\alpha_{1} ; \varphi} h_{u}(b)-\mathcal{I}_{0^{+}}^{\alpha_{1} ; \varphi} h_{v}(b)\right) \mid \\
& \leq(q-1) y^{q-2}\left(\frac{2 M^{\alpha_{1}}}{\Gamma\left(\alpha_{1}+1\right)} \sup _{t \in[a, b]}\left|h_{u}(t)-h_{v}(t)\right|+\frac{M^{\rho}}{\Gamma(\rho+1)} \sup _{t \in[a, b]}|u(t)-v(t)|\right) \\
& \leq(q-1) y^{q-2}\left(\left(\frac{2 \Upsilon M^{\alpha_{1}}}{\Gamma\left(\alpha_{1}+1\right)}+\frac{M^{\rho}}{\Gamma(\rho+1)}\right) \sup _{t \in[a, b]}|u(t)-v(t)|\right. \\
& \left.+\frac{2 \Upsilon M^{\alpha_{1}}}{\Gamma\left(\alpha_{1}+1\right)} \sup _{t \in[a, b]}\left|R L \mathcal{D}_{a^{+}}^{\mu ; \varphi} u(t)-{ }^{R L} \mathcal{D}_{a^{+}}^{\mu ; \varphi} v(t)\right|\right) \\
& \leq(q-1) \Delta^{q-2}\left(\frac{4 \Upsilon M^{\alpha_{1}}}{\Gamma\left(\alpha_{1}+1\right)}+\frac{M^{\rho}}{\Gamma(\rho+1)}\right)\|u-v\|_{C_{\varphi}^{\mu}} \text {. }
\end{aligned}
$$

where

$$
\left\{\begin{array}{c}
\Delta>\frac{2 \Upsilon M^{\alpha_{1}}}{\Gamma\left(\alpha_{1}+1\right)}+\frac{M^{\rho}}{\Gamma(\rho+1)}, \text { if } q>2 \\
\text { ou } \\
0<\Delta \leq \frac{2 \Upsilon M^{\alpha_{1}}}{\Gamma\left(\alpha_{1}+1\right)}+\frac{M^{\rho}}{\Gamma(\rho+1)}, \text { if } 1<q \leq 2
\end{array}\right.
$$

Then

$$
\begin{aligned}
& \sup _{t \in[a, b]}|(\mathbb{G} u)(t)-(\mathbb{G} v)(t)| \\
& \leq \sup _{t \in[a, b]}\left|\frac{1}{\Gamma\left(\alpha_{2}\right)} \int_{a}^{t} \varphi^{\prime}(s) \varphi_{\alpha_{2}-1}(t, s)\left(X_{u}-X_{v}\right)(s, a) d s\right| \\
& +\sup _{t \in[a, b]}\left|\frac{\varphi_{\gamma_{2}-1}(t, a)}{\Gamma\left(\alpha_{2}\right) \varphi_{\gamma_{2}-1}(b, a)} \int_{a}^{b} \varphi^{\prime}(t) \varphi_{\alpha_{2}-1}(b, t)\left(X_{u}-X_{v}\right)(t, a) d t\right| \\
& +\sup _{t \in[a, b]}\left|\frac{\varphi_{\gamma_{2}-1}(t, a)}{\varphi_{\gamma_{2}-1}(b, a)} \sum_{i=1}^{n} \lambda_{i}\left(u\left(\zeta_{i}\right)-v\left(\zeta_{i}\right)\right)\right|
\end{aligned}
$$




$$
\begin{aligned}
& \leq \frac{2 M^{\alpha_{2}}}{\Gamma\left(\alpha_{2}+1\right)} \sup _{t \in[a, b]}\left|X_{u}(t, a)-X_{v}(t, a)\right|+\Lambda_{2} \sup _{t \in[a, b]}(|u(t)-v(t)|) \\
& \leq\left(\frac{2(q-1) \Delta^{q-2} M^{\alpha_{2}}}{\Gamma\left(\alpha_{2}+1\right)}\left(\frac{4 \Upsilon M^{\alpha_{1}}}{\Gamma\left(\alpha_{1}+1\right)}+\frac{M^{\rho}}{\Gamma(\rho+1)}\right)+\Lambda_{2}\right)\|u-v\|_{C_{\varphi}^{\mu}} \\
& \leq \Upsilon_{1}\|u-v\|_{C_{\varphi}^{\mu}} .
\end{aligned}
$$

Also

$$
\begin{aligned}
& \sup _{t \in[a, b]}||\left({ }^{R L} \mathcal{D}_{a^{+}}^{\mu ; \varphi} \mathbb{G} u\right)(t)-\left({ }^{R L} \mathcal{D}_{a^{+}}^{\mu ; \varphi} \mathbb{G} v\right)(t) \mid \\
\leq & \sup _{t \in[a, b]} \mid \frac{1}{\Gamma\left(\alpha_{2}-\mu\right)} \int_{a}^{t} \varphi^{\prime}(s) \varphi_{\alpha_{2}-\mu-1}(t, s)\left(X_{u}-X_{v}\right)(s, a) d s \\
& +\frac{\Gamma\left(\gamma_{2}\right)}{\Gamma\left(\gamma_{2}-\mu\right)} \frac{\varphi_{\gamma_{2}-\mu-1}(t, a)}{\varphi_{\gamma_{2}-1}(b, a)} \sum_{i=1}^{n} \lambda_{i}\left(u\left(\zeta_{i}\right)-v\left(\zeta_{i}\right)\right) \\
& +\frac{\Gamma\left(\gamma_{2}\right)}{\Gamma\left(\alpha_{2}\right) \Gamma\left(\gamma_{2}-\mu\right)} \frac{\varphi_{\gamma_{2}-\mu-1}(t, a)}{\varphi_{\gamma_{2}-1}(b, a)} \int_{a}^{b} \varphi^{\prime}(t) \varphi_{\alpha_{2}-1}(b, t)\left(X_{u}-X_{v}\right)(t, a) d t \mid \\
\leq & \left(\frac{M^{\alpha_{2}-\mu}}{\Gamma\left(\alpha_{2}-\mu+1\right)}+\frac{\Gamma\left(\gamma_{2}\right) M^{\alpha_{2}-\mu}}{\Gamma\left(\alpha_{2}+1\right) \Gamma\left(\gamma_{2}-\mu\right)}\right) \sup _{t \in[a, b]}\left|X_{u}-X_{v}\right| \\
& +\frac{\Gamma\left(\gamma_{2}\right) \Lambda_{2} M^{-\mu}}{\Gamma\left(\gamma_{2}-\mu\right)} \sup _{t \in[a, b]}(|u(t)-v(t)|) \\
\leq & \left\{(q-1) \Delta^{q-2}\left(\frac{4 \Upsilon M^{\alpha_{1}}}{\Gamma\left(\alpha_{1}+1\right)}+\frac{M^{\rho}}{\Gamma(\rho+1)}\right)\left(\frac{M^{\alpha_{2}-\mu}}{\Gamma\left(\alpha_{2}-\mu+1\right)}+\frac{\Gamma\left(\gamma_{2}\right) M^{\alpha_{2}-\mu}}{\Gamma\left(\alpha_{2}+1\right) \Gamma\left(\gamma_{2}-\mu\right)}\right)\right. \\
& \left.+\frac{\Gamma\left(\gamma_{2}\right) \Lambda_{2} M^{-\mu}}{\Gamma\left(\gamma_{2}-\mu\right)}\right\}\|u-v\|_{C_{\varphi}^{\mu}} \\
\leq & \Upsilon_{2}\|u-v\|_{C_{\varphi}^{\mu}} \cdot
\end{aligned}
$$

By (3.4) and (3.5), yields the following inequality

$$
\|\mathbb{G} u-\mathbb{G} v\|_{C_{\varphi}^{\mu}} \leq\left(\Upsilon_{1}+\Upsilon_{2}\right)\|u-v\|_{C_{\varphi}^{\mu}}
$$

Where $\Upsilon_{1}+\Upsilon_{2}<1$. Hence $\mathbb{G}$ is a contraction operator and the contraction mapping principle implies that (1.1) has a unique solution.

3.3. Ulam Type Stability. We introduce the following two definitions

Definition 4. The problem (1.1) is Ulam-Hyers stable if $\exists \lambda \in \mathbb{R}_{+}^{*}$, such that for each $\varepsilon>0, t \in J$, and for each $u \in C_{\varphi}^{\mu}$ solution of the following inequality

$$
\left\|{ }^{H} \mathcal{D}_{a^{+}}^{\alpha_{1}, \beta_{1} ; \varphi} \psi_{p}\left({ }^{H} \mathcal{D}_{a^{+}}^{\alpha_{2}, \beta_{2} ; \varphi} u\right)(t)-h\left(t, u(t),{ }^{R L} \mathcal{D}_{a^{+}}^{\mu ; \varphi} u(t)\right)\right\|_{C_{\varphi}^{\mu}}<\varepsilon,
$$

$\exists v \in C_{\varphi}^{\mu}$ solution of (1.1), i.e.

$$
{ }^{H} \mathcal{D}_{a^{+}}^{\alpha_{1}, \beta_{1} ; \varphi} \psi_{p}\left({ }^{H} \mathcal{D}_{a^{+}}^{\alpha_{2}, \beta_{2} ; \varphi} v\right)(t)=h\left(t, v(t),{ }^{R L} \mathcal{D}_{a^{+}}^{\mu ; \varphi} v(t)\right),
$$


such that, the inequality

$$
\|u-v\|_{C_{\varphi}^{\mu}} \leq \lambda \varepsilon
$$

holds.

Definition 5. The equation (1.1) has the Ulam-Hyers stability in the generalized sense if $\exists \varphi \in$ $C\left(J, \mathbb{R}_{+}\right)$, such that for each $\varepsilon>0, t \in J$, and for each $u \in C_{\varphi}^{\mu}$ solution of:

$$
\left\|{ }^{H} \mathcal{D}_{a^{+}}^{\alpha_{1}, \beta_{1} ; \varphi} \psi_{p}\left({ }^{H} \mathcal{D}_{a^{+}}^{\alpha_{2}, \beta_{2} ; \varphi} u\right)(t)-h\left(t, u(t),{ }^{R L} \mathcal{D}_{a^{+}}^{\mu ; \varphi} u(t)\right)\right\|_{C_{\varphi}^{\mu}}<\varepsilon,
$$

$\exists v \in C_{\varphi}^{\mu}$ solution of (1.1) that satisfies

$$
\|u(t)-v(t)\|_{C_{\varphi}^{\mu}} \leq \varepsilon \varphi(t)
$$

In the light of the first definition and using the above existence and uniqueness theorem, we present to the reader the following result.

Theorem 3. If the assumptions $\left(\mathcal{H}_{2}\right)$ are satisfied, then Eq (1.1) is Ulam-Hyers stable under the condition that $N_{1}+N_{2}<1$, where

$$
N_{1}=\frac{2(q-1) \Delta^{q-2} M^{\alpha_{2}}}{\Gamma\left(\alpha_{2}+1\right)}\left(\frac{4 \Upsilon M^{\alpha_{1}}}{\Gamma\left(\alpha_{1}+1\right)}+\frac{M^{\rho}}{\Gamma(\rho+1)}\right)
$$

and

$$
N_{2}=\left(\frac{4 \Upsilon(q-1) \Delta^{q-2} M^{\alpha_{1}}}{\Gamma\left(\alpha_{1}+1\right)}+\frac{(q-1) \Delta^{q-2} M^{\rho}}{\Gamma(\rho+1)}\right)\left(\frac{M^{\alpha_{2}-\mu}}{\Gamma\left(\alpha_{2}-\mu+1\right)}+\frac{\Gamma\left(\gamma_{2}\right) M^{\alpha_{2}-\mu}}{\Gamma\left(\alpha_{2}+1\right) \Gamma\left(\gamma_{2}-\mu\right)}\right) .
$$

Proof. Let $u \in C_{\varphi}^{\mu}$ be a solution of the inequality (3.6), i.e.

$$
\left\|{ }^{H} \mathcal{D}_{a^{+}}^{\alpha_{1}, \beta_{1} ; \varphi} \psi_{p}\left({ }^{H} \mathcal{D}_{a^{+}}^{\alpha_{2}, \beta_{2} ; \varphi} u\right)(t)-h\left(t, u(t),{ }^{R L} \mathcal{D}_{a^{+}}^{\mu ; \varphi} u(t)\right)\right\|_{C_{\varphi}^{\mu}}<\varepsilon, \quad \forall t \in J .
$$

Let $v \in C_{\varphi}^{\mu}$ be a unique solution of:

$$
{ }^{H} \mathcal{D}_{a^{+}}^{\alpha_{1}, \beta_{1} ; \varphi} \psi_{p}\left({ }^{H} \mathcal{D}_{a^{+}}^{\alpha_{2}, \beta_{2} ; \varphi} v\right)(t)=h\left(t, v(t),{ }^{R L} \mathcal{D}_{a^{+}}^{\mu ; \varphi} v(t)\right), \quad \forall t \in J
$$

and

$$
\left\{\begin{array}{c}
u(a)=v(a), u(b)=v(b) \\
\text { and } \\
\psi_{p}\left({ }^{H} \mathcal{D}_{a^{+}, \beta_{2} ; \varphi}^{\alpha_{2}} u\right)(a)=\psi_{p}\left({ }^{H} \mathcal{D}_{a^{+}, \beta_{2} ; \varphi}^{\alpha_{2}} v\right)(a) \\
\psi_{p}\left({ }^{H} \mathcal{D}_{a^{+}}^{\alpha_{2}, \beta_{2} ; \varphi} u\right)(b)=\psi_{p}\left({ }^{H} \mathcal{D}_{a^{+}}^{\alpha_{2}, \beta_{2} ; \varphi} v\right)(b),
\end{array}\right.
$$


By using Proof of Lemma 6

$$
\begin{aligned}
v(t)= & \frac{1}{\Gamma\left(\alpha_{2}\right)} \int_{a}^{t} \varphi^{\prime}(s) \varphi_{\alpha_{2}-1}(t, s) X_{v}(s, a) d s \\
& -\frac{\varphi_{\gamma_{2}-1}(t, a)}{\Gamma\left(\alpha_{2}\right) \varphi_{\gamma_{2}-1}(b, a)} \int_{a}^{b} \varphi^{\prime}(t) \varphi_{\alpha_{2}-1}(b, t) X_{v}(t, a) d t \\
& +\frac{\varphi_{\gamma_{2}-1}(t, a)}{\varphi_{\gamma_{2}-1}(b, a)} \sum_{i=1}^{n} \lambda_{i} u\left(\zeta_{i}\right),
\end{aligned}
$$

where

$$
X_{v}(s, a)=\psi_{q}\left(\frac{1}{\Gamma\left(\alpha_{1}\right)} \int_{a}^{s} \varphi^{\prime}(s) \varphi_{\alpha_{1}-1}(s, z) h_{v}(z) d z+\frac{\left(\mathcal{I}_{0^{+}}^{\rho ; \varphi} u(\zeta)-\mathcal{I}_{0^{+}}^{\alpha_{1} ; \varphi} h_{v}(b)\right)}{\varphi_{\gamma_{1}-1}(b, a)} \varphi_{\gamma_{1}-1}(s, a)\right)
$$

By integration of inequality (3.9), for any $t \in J$, we have

$$
\begin{aligned}
& \| u(t)-\frac{1}{\Gamma\left(\alpha_{2}\right)} \int_{a}^{t} \varphi^{\prime}(s) \varphi_{\alpha_{2}-1}(t, s) X_{u}(s, a) d s \\
& +\frac{\varphi_{\gamma_{2}-1}(t, a)}{\Gamma\left(\alpha_{2}\right) \varphi_{\gamma_{2}-1}(b, a)} \int_{a}^{b} \varphi^{\prime}(t) \varphi_{\alpha_{2}-1}(b, t) X_{u}(t, a) d t \\
& \quad-\frac{\varphi_{\gamma_{2}-1}(t, a)}{\varphi_{\gamma_{2}-1}(b, a)} \sum_{i=1}^{n} \lambda_{i} u\left(\zeta_{i}\right) \|_{C} \\
& \leq \mathcal{I}_{a^{+}}^{\alpha_{2} ; \varphi} \psi_{q}\left(\mathcal{I}_{a^{+}}^{\alpha_{1} ; \varphi} \varepsilon\right)=\frac{M^{q-1} \varphi_{\alpha_{1}+\alpha_{2}}(t, a)}{\Gamma\left(\alpha_{1}+\alpha_{2}+1\right)} \varepsilon .
\end{aligned}
$$

On the other hand, for any $u, v \in C_{\varphi}^{\mu}$, we have the following estimate

$$
\begin{aligned}
& \|u(t)-v(t)\|_{C} \\
< & \frac{M^{q-1} \varphi_{\alpha_{1}+\alpha_{2}}(t, a)}{\Gamma\left(\alpha_{1}+\alpha_{2}+1\right)} \varepsilon \\
& +\sup _{t \in[a, b]}\left|\frac{1}{\Gamma\left(\alpha_{2}\right)} \int_{a}^{t} \varphi^{\prime}(s) \varphi_{\alpha_{2}-1}(t, s)\left(X_{u}-X_{v}\right)(s, a) d s\right| \\
& +\sup _{t \in[a, b]}\left|\frac{\varphi_{\gamma_{2}-1}(t, a)}{\Gamma\left(\alpha_{2}\right) \varphi_{\gamma_{2}-1}(b, a)} \int_{a}^{b} \varphi^{\prime}(t) \varphi_{\alpha_{2}-1}(b, t)\left(X_{u}-X_{v}\right)(t, a) d t\right| \\
< & \frac{M^{\alpha_{1}+\alpha_{2}+q 1}}{\Gamma\left(\alpha_{1}+\alpha_{2}+1\right)} \varepsilon+\frac{2(q-1) \Delta^{q-2} M^{\alpha_{2}}}{\Gamma\left(\alpha_{2}+1\right)}\left(\frac{4 \Upsilon M^{\alpha_{1}}}{\Gamma\left(\alpha_{1}+1\right)}+\frac{M^{\rho}}{\Gamma(\rho+1)}\right)\|u-v\|_{C_{\varphi}^{\mu}} \\
< & \frac{M^{\alpha_{1}+\alpha_{2}+q-1}}{\Gamma\left(\alpha_{1}+\alpha_{2}+1\right)} \varepsilon+N_{1}\|u-v\|_{C_{\varphi}^{\mu}} .
\end{aligned}
$$


Also, for any $t \in J$, we have

$$
\begin{aligned}
& \left\|^{R L} \mathcal{D}_{a^{+}}^{\mu ; \varphi}(u(t)-v(t))\right\|_{C} \\
\leq & \frac{M^{q-1} \varphi_{\alpha_{1}+\alpha_{2}-\mu}(t, a)}{\Gamma\left(\alpha_{1}+\alpha_{2}-\mu+1\right)} \varepsilon \\
& +\sup _{t \in[a, b]} \mid \frac{1}{\Gamma\left(\alpha_{2}-\mu\right)} \int_{a}^{t} \varphi^{\prime}(s) \varphi_{\alpha_{2}-\mu-1}(t, s)\left(X_{u}-X_{v}\right)(s, a) d s \\
& +\frac{\Gamma\left(\gamma_{2}\right)}{\Gamma\left(\alpha_{2}\right) \Gamma\left(\gamma_{2}-\mu\right)} \frac{\varphi_{\gamma_{2}-\mu-1}(t, a)}{\varphi_{\gamma_{2}-1}(b, a)} \int_{a}^{b} \varphi^{\prime}(t) \varphi_{\alpha_{2}-1}(b, t)\left(X_{u}-X_{v}\right)(t, a) d t \mid \\
\leq & \frac{M^{\alpha_{1}+\alpha_{2}+q-\mu-1}}{\Gamma\left(\alpha_{1}+\alpha_{2}-\mu+1\right)} \varepsilon+\left(\frac{M^{\alpha_{2}-\mu}}{\Gamma\left(\alpha_{2}-\mu+1\right)}+\frac{\Gamma\left(\gamma_{2}\right) M^{\alpha_{2}-\mu}}{\Gamma\left(\alpha_{2}+1\right) \Gamma\left(\gamma_{2}-\mu\right)}\right) \sup _{t \in[a, b]}\left|X_{u}-X_{v}\right| \\
\leq & \frac{M^{\alpha_{1}+\alpha_{2}+q-\mu-1}}{\Gamma\left(\alpha_{1}+\alpha_{2}-\mu+1\right)} \varepsilon \\
& +\left(\frac{4 \Upsilon(q-1) \Delta{ }^{q-2} M^{\alpha_{1}}}{\Gamma\left(\alpha_{1}+1\right)}+\frac{(q-1) \Delta^{q-2} M^{\rho}}{\Gamma(\rho+1)}\right)\left(\frac{M^{\alpha_{2}-\mu}}{\Gamma\left(\alpha_{2}-\mu+1\right)}+\frac{\Gamma\left(\gamma_{2}\right) M^{\alpha_{2}-\mu}}{\Gamma\left(\alpha_{2}+1\right) \Gamma\left(\gamma_{2}-\mu\right)}\right)\|u-v\|_{C_{\varphi}^{\mu}}^{\mu} \\
\leq & \frac{M^{\alpha_{1}+\alpha_{2}+q-\mu-1}}{\Gamma\left(\alpha_{1}+\alpha_{2}-\mu+1\right)} \varepsilon+N_{2}\|u-v\|_{C_{\varphi}^{\mu} .}
\end{aligned}
$$

So, by (3.11) and (3.12) we have

$$
\|u-v\|_{C_{\varphi}^{\mu}} \leq \varepsilon\left(\frac{M^{\alpha_{1}+\alpha_{2}+q-1}}{\Gamma\left(\alpha_{1}+\alpha_{2}+1\right)}+\frac{M^{\alpha_{1}+\alpha_{2}+q-\mu-1}}{\Gamma\left(\alpha_{1}+\alpha_{2}-\mu+1\right)}\right)+\left(N_{1}+N_{2}\right)\|u-v\|_{C_{\varphi}^{\mu}} .
$$

Therefore, we get

$$
\|u-v\|_{C_{\varphi}^{\mu}} \leq \lambda \varepsilon
$$

such that

$$
\lambda=\frac{1}{1-\left(N_{1}+N_{2}\right)}\left(\frac{M^{\alpha_{1}+\alpha_{2}+q-1}}{\Gamma\left(\alpha_{1}+\alpha_{2}+1\right)}+\frac{M^{\alpha_{1}+\alpha_{2}+q-\mu-1}}{\Gamma\left(\alpha_{1}+\alpha_{2}-\mu+1\right)}\right),
$$

for any $t \in J$. This implies that the Ulam-Hyers stability condition is satisfied.

\subsection{Illustrative exemple. Consider the following problem}

$$
\begin{gathered}
{ }^{H} \mathcal{D}_{0^{+}}^{\frac{13}{10}, \frac{6}{7} ; t^{2}} \psi_{p}\left(\left({ }^{H} \mathcal{D}_{0^{+}}^{\frac{17}{10}, \frac{2}{3} ; t^{2}} u\right)\right)(t)=h\left(t, u(t),{ }^{R L} \mathcal{D}_{a^{+}}^{\frac{1}{2} ; t^{2}} u(t)\right), t \in J=[0,2], \\
u(a)=0, u(2)=\sum_{i=1}^{n}\left(\frac{3 i}{11}\right) u\left(\frac{i}{2+i}\right), \\
\psi_{p}\left(\left({ }^{H} \mathcal{D}_{0^{+}}^{\frac{17}{10}, \frac{2}{3} ; t^{2}} u\right)\right)(0)=0, \psi_{p}\left(\left({ }^{H} \mathcal{D}_{0^{+}}^{\frac{17}{10}, \frac{2}{3} ; t^{2}} u\right)\right)(2)=\mathcal{I}_{a^{+}}^{\frac{3}{2} ; t^{2}} u\left(\frac{4}{3}\right) \\
f(t, u(t), v(t))=\exp \left(\frac{1}{7\left(1+t^{2}\right)}\right) u(t)+\frac{v(t)}{\left(1+e^{t}\right)},
\end{gathered}
$$


then assumptions $\left(\mathcal{H}_{1}\right),\left(\mathcal{H}_{2}\right)$ and $\left(\mathcal{H}_{3}\right)$ are satisfied with

$$
\Upsilon=\pi_{2}^{*}=\frac{1}{2}, \pi_{1}^{*}=e^{\frac{1}{7}} \text {, and } M=4
$$

We conclude that (3.13) has an unique solution.

\section{REFERENCES}

[1] B. Ahmad, A. Alsaedi, S. K. Ntouyas, and J. Tariboon, Hadamard-Type Fractional Differential Equations, Inclusions and Inequalities, Springer, Cham, Switzerland, 2017.

[2] S. Asawasamrit, A. Kijjathanakorn, S. K. Ntouyas, and J. Tariboon, Nonlocal boundary value problems for Hilfer fractional differential equations, Bull. Korean Math. Soc. 55 (2018) 1639-1657. https://doi .org/10.4134/BKMS . b170887.

[3] H. Beddani, $n+1$-Parameter singular fractional differential equation, Asia Math. 5 (2021) 11-18. http://dx. doi.org/10.5281/zenodo.4721390.

[4] H. Beddani, Z. Dahmani, Solvability for a nonlinear differential problem of Langevin type via phi-Caputo approach, Eur. J. Math. Appl. 1 (2021), 11. https://doi.org/10.28919/ejma.2021.1.11.

[5] H. Beddani, Z. Dahmani, I. Jebril, A Sequential random pronlem of airy type solved by the lower and upper method, ROMAI J. 16 (2020) 37-49

[6] H. Beddani, M. Beddani, Solvability for a differential systems via Phi-Caputo approach, J. Sci. Arts, 3(56) (2021).

[7] A. Devi, A. Kumar, D. Baleanu and A. Khan, On stability analysis and existence of positive solutions for a general non-linear fractional differential equations. Adv. Differ. Equations 2020 (2020) 300. https://doi.org/10.1186/ s13662-020-02729-3.

[8] K. Diethelm, The Analysis of Fractional Differential Equations, Lecture Notes in Mathematics, Springer, New York, 2010.

[9] K. M. Furati, N. D. Kassim, and N. E. Tatar, Existence and uniqueness for a problem involving Hilfer fractional derivative, Comput. Math. Appl. 64 (2012), 1616-1626. https://doi.org/10.1016/ j. camwa. 2012.01.009.

[10] H. Gu and J. J. Trujillo, Existence of mild solution for evolution equation with Hilfer fractional derivative, Appl. Math. Comput. 257 (2015) 344-354. https://doi.org/10.1016/j. amc.2014.10.083.

[11] R. Hilfer, Applications of Fractional Calculus in Physics, World Scientific, Singapore, 2000.

[12] R. Hilfer, Experimental evidence for fractional time evolution in glass forming materials, J. Chem. Phys. 284 (2002) 399-408. https://doi.org/10.1016/S0301-0104(02)00670-5.

[13] R. Hilfer, Y. Luchko, and Z. Tomovski, Operational method for the solution of fractional differential equations with generalized Riemann-Liouville fractional derivatives, Frac. Calc. Appl. Anal. 12 (2009) 299-318.

[14] H. Khan, W. Chen, H. Sun, Analysis of positive solution and Hyers-Ulam stability for a class of singular fractional differential equations with p-Laplacian in Banach space. Math. Methods Appl. Sci. 41 (2018) 3430-3440. https: //doi.org/10.1002/mma.4835.

[15] A. Khan, M.I. Syam, A.,Zada, H. Khan, : Stability analysis of nonlinear fractional differential equations with Caputo and Riemann-Liouville derivatives. Eur. Phys. J. Plus 133, 26 (2018). https://doi.org/10.1140/epjp/ i2018-12119-6.

[16] H. Khan, T. Abdeljawad, M. Aslam, R. A. Khan and A. Khan, Existence of positive solution and Hyers-Ulam stability for a nonlinear singular-delay-fractional differential equation. Adv. Differ. Equations 2019 (2019) 104. https://doi.org/10.1186/s13662-019-2054-z.

[17] A. A. Kilbas, H. M. Srivastava, and J. J. Trujillo, Theory and Applications of the Fractional Differential Equations, North-Holland Mathematics Studies, vol. 204, Elsevier, Amsterdam, 2006. 
[18] V. Lakshmikantham, S. Leela, and J. V. Devi, Theory of Fractional Dynamic Systems, Cambridge Scientific Publishers, Cambridge, 2009.

[19] Y. Li, Existence of positive solutions for fractional differential equation involving integral boundary conditions with p- Laplacian operator. Adv. Differ. Equ. 2017 (2017) 135. https://doi.org/10.1186/s13662-017-1172-8.

[20] K. S. Miller and B. Ross, An Introduction to the Fractional Calculus and Differential Equations, John Wiley, New York, 1993.

[21] C. Nuchpong, S.K. Ntouyas, and J. Tariboon,. worked on Boundary value problems of Hilfer-type fractional integrodifferential equations and inclusions with nonlocal integro-multipoint boundary conditions, Open Math. 18 (2020) 1879-1894. https://doi.org/10.1515/math-2020-0122

[22] I. Podlubny, Fractional Differential Equations, Academic Press, New York, 1999.

[23] S. G. Samko, A. A. Kilbas, and O. I. Marichev, Fractional Integrals and Derivatives, Gordon and Breach Science, Yverdon, 1993

[24] A. Seemab, J. Alzabut, M. Rehman, Y. Adjabi, M.S.Abdo, Langevin equation with nonlocal boundary conditions involving a $\varphi$-Caputo fractional operator: arXiv:2006.00391v1 [math.AP] 31 May 2020.

[25] C. Vanterler da, J. Sousa, ; Capelas de Oliveira, E. On the $\varphi$-Hilfer fractional derivative. Commun. Nonlinear Sci. Numer. Simul. 60 (2018) 72-91.

[26] J. Wang and Y. Zhang, Nonlocal initial value problems for differential equations with Hilfer fractional derivative, Appl. Math. Comput. 266 (2015), 850-859. https://doi.org/10.1016/j . amc. 2015.05.144.

[27] Y. Wang, Existence and nonexistence of positive solutions for mixed fractional boundary value problem with parameter and p-Laplacian operator. J. Funct. Spaces 2018 (2018) Article ID 1462825. https://doi.org/10.1155/2018/ 1462825.

[28] A. Wongcharoen, B. Ahmad, S. K. Ntouyas, and J. Tariboon, Three-point boundary value problems for Langevin equation with Hilfer fractional derivative, Adv. Math. Phys. 2020 (2020), 9606428. https://doi.org/10.1155/ $2020 / 9606428$.

[29] A. Wongcharoen, S. K. Ntouyas, and J. Tariboon, Nonlocal boundary value problems for Hilfer type pantograph fractional differential equations and inclusions, Adv. Differ. Equ. 2020 (2020), 279. https://doi.org/10.1186/ s13662-020-02747-1.

[30] A. Wongcharoen, S. K. Ntouyas, and J. Tariboon, Boundary value problems for Hilfer fractional differential inclusions with nonlocal integral boundary conditions, Mathematics 8 (2020), 1905. https://doi.org/10.3390/ math8111905.

[31] Y. Zhou, Basic Theory of Fractional Differential Equations, World Scientific, Singapore, 2014. 